\title{
NMR studies of methane and hydrogen in microporous materials
}

\author{
Yu Ji, J.A. Hamida, Yibing Tang, and N.S. Sullivan \\ Department of Physics, University of Florida, Gainesville, FL, USA \\ E-mail: sullivan@phys.ufl.edu
}

Received September 7, 2015, published online December 23, 2015

\begin{abstract}
We review the results of nuclear magnetic resonance studies of the molecular dynamics of the quantum gases $\mathrm{HD}$ and $\mathrm{CH}_{4}$ adsorbed in the cages of microporous structures. Measurements of the variation of the nuclear spinlattice and nuclear spin-spin relaxation times with temperature provide detailed information about the translational and rotational dynamics of the adsorbed molecules over a wide temperature range.
\end{abstract}

PACS: 76.60.-k Nuclear magnetic resonance and relaxation.

Keywords: magnetic resonance, microporous materials, quantum gases $\mathrm{HD}$ and $\mathrm{CH}_{4}$.

\section{Introduction}

The absorption and dynamics of $\mathrm{H}_{2}$ and $\mathrm{CH}_{4}$ among other light gases $\left(\mathrm{CO}, \mathrm{CO}_{2}, \mathrm{~N}_{2} \ldots\right)$ when confined to the internal cages of mesoporous structures has received great interest in recent years because of the potential use of these mesoporous materials (MOFs, MCM, zeolites...) for the storage and transport of commercially important gases [1-7]. The efficiencies of various nanoporous and microporous materials for storage and ease of removal depend crucially on the thermodynamic properties of these gases at or near the surfaces of the chosen materials [8-12]. These properties and the characteristic dynamics are also important in the development of methods for $\mathrm{CO}_{2}$ sequestration [13-15]. There is considerable documentation in the literature about the total absorption properties of light gases (such as hydrogen and methane) on various porous materials but there is little information about the detailed dynamics of the gases in the confined geometries or about their interactions with the walls of the confinement. One of the great advantages of the use of NMR techniques is that they can address this need. In this paper we review recent applications of NMR methods to study the dynamics of $\mathrm{CH}_{4}$ and HD in microporous materials [16-19].

\section{Experimental methods}

Careful NMR techniques can be very powerful tools for measuring the dynamics of molecules in condensed matter systems. The motion of the molecules modulates the nuclear dipole-dipole interactions between the spins of the molecules as well as the interactions between the spins of the mobile molecules and the magnetic spins in the walls

(c) Yu Ji, J.A. Hamida, Yibing Tang, and N.S. Sullivan, 2016 of the porous adsorbers. The Fourier components of these fluctuations at $\omega=\omega_{L}$, the nuclear Larmor frequency, can induce transitions between the nuclear spin levels of the molecules and this will determine the nuclear spin-lattice relaxation times. In order to calculate the relaxation times we follow Cowan [20] and express the dipolar spin-spin interactions in terms of irreducible components

$$
H_{\text {dip }}=\sum_{m=-2}^{2} D_{m}
$$

where for like spins

$$
D_{m}=\frac{\mu_{0}}{4 \pi} \frac{\hbar^{2} \gamma^{4}}{\sqrt{5}} \sum_{i j} Y_{2}^{-m}\left(\Omega_{i j}\right) T_{2}^{m}(i j) / r_{i j}^{3},
$$

$\mu_{0}$ is the permeability of free space, $\gamma$ is the nuclear gyromagnetic ratio of the nucleus, $Y_{2}^{m}\left(\Omega_{i j}\right)$ is the spherical harmonic for the angles $\Omega_{i j}=\left(\theta_{i j}, \varphi_{i j}\right)$ specifying the orientation of the vector $r_{i j}$ with respect to the applied magnetic field, $\theta_{i j}$ is the polar angle and $\varphi_{i j}$ is the azimuthal angle for the vector $r_{i j}$. The $T_{2}^{m}(i j)$ are irreducible nuclear spin operators that transform under rotation in the same manner as the spherical harmonics:

$$
\begin{gathered}
T_{2}^{0}(i j)=I_{i} \cdot I_{j}-3 I_{i}^{z} I_{j}^{z}, \\
T_{2}^{1}(i j)=\sqrt{\frac{3}{2}}\left[I_{i}^{z} I_{j}^{+}+I_{i}^{+} I_{j}^{z}\right], \\
T_{2}^{2}(i j)=-\sqrt{\frac{3}{2}} I_{i}^{+} I_{j}^{+},
\end{gathered}
$$

with $T_{2}^{-m}(i j)=(-)^{m}\left[T_{2}^{m}(i j)\right]^{\dagger}$. 
In the simplest cases where a single correlation time for the fluctuations dominates one can obtain a direct measure of the microscopic motion:

$$
\frac{1}{T_{1}}=\frac{2}{3} M_{2} \sum_{n=1,2} n^{2} J_{n}\left(n \omega_{L}\right),
$$

where $M_{2}$ is the second moment of the nuclear spin interactions and $J_{n}(\omega)$ is the Fourier component of the autocorrelation functions for the dipolar interactions given by $G_{n}(t)=\left\langle Y_{2 n}^{*}(t) Y_{2 n}(0)\right\rangle$.

This simple case often occurs for the translational and rotational diffusion of molecules in condensed phases. If the motion is characterized by a unique correlation time, $\tau_{c}$, as for example, given by the microscopic jump time from one lattice site to a neighboring site in the case of translational diffusion, the NMR measurements can determine the correlation time and thus the diffusion constant ( $D \sim z a_{0}^{2} \tau_{c}^{-1}$, where $a_{0}$ is the lattice spacing and $z$ the coordination number). These times inferred from NMR measurements can be compared with those deduced from neutron scattering measurements. In addition to the spinlattice relaxation time one can also measure the nuclear spin-spin relaxation times, $T_{2}$. These times are determined by the Fourier component at close to $\omega=0$. Specifically,

$$
\frac{1}{T_{2}}=\frac{1}{4} \sum_{n=-2}^{2}\left(6-n^{2}\right) J_{n}\left(n \omega_{L}\right) .
$$

In the case of a unique correlation time for the motion, as is often the case for motions associated with quantum jumping in adsorbed gases we can simplify Eqs. (6) and (7). For rapid motion where the correlation time $\tau_{c}<<\omega_{L}^{-1}$ we have

$$
\frac{1}{T_{1}}=\frac{1}{T_{2}}=\frac{10}{3} M_{2} \tau_{c} .
$$

In the opposite case of slow motions, for which $\tau_{c}<<\omega_{L}$, we have

$$
\begin{gathered}
\frac{1}{T_{1}}=\frac{4}{3} \frac{M_{2}}{\omega_{L}^{2} \tau_{c}}, \\
\frac{1}{T_{2}}=M_{2} \tau_{c} .
\end{gathered}
$$

In many cases at low temperatures there can be several sources of excitations; phonons, spin-interactions, quantum diffusion, and the different interactions can form weakly coupled energy reservoirs. In this situation the relaxation will depend on the couplings between the respective energy baths associated with these excitations as illustrated in Fig. 1 where $R_{X Y}$ represents the relaxation rate between baths $X$ and $Y$.

If the energy baths can attain thermal equilibrium in a time short compared to the relaxation couplings between

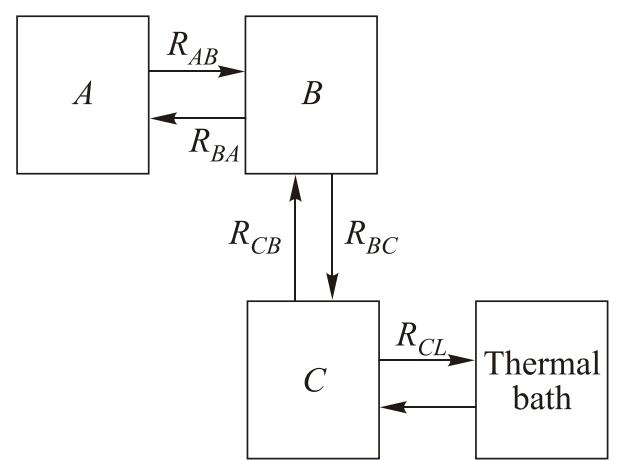

Fig. 1. Illustration of different weakly coupled energy baths designated by $A, B$ and $C$ where the weakest coupling is the vertical path between $B$ and $C$.

the baths, the relaxation time observed experimentally will vary with changes in the heat capacities of the individual baths. In Fig. 1 we show the case where the coupling $R_{B C}$ is the slowest (or weakest) connection for the energy flow. In this case the observed relaxation time will be

$$
T_{1, \text { observed }}=\frac{C_{A}+C_{B}}{C_{B}} T_{B C},
$$

where $C_{A}, C_{B}$ are the heat capacities of baths $A$ and $B$. When this situation occurs, the experiments often show a two-stage relaxation process: (i) a short-time relaxation as an energy bath comes into internal thermal equilibrium after a disturbance, and (ii) a long-time component to the relaxation determined by cross-relaxation between different energy baths.

\section{Experimental observations}

Prior to the NMR studies the mesoporous substrate materials were all first characterized by measuring the adsorption isotherm at a fixed temperature. A typical example for methane adsorbed on zeolite is shown in Fig. 2 for a sample maintained at $77 \mathrm{~K}$. Small gas volumes are adsorbed sequentially and the equilibrium pressure at each step is recorded as one point on the graph. The observation of at least two steps in the isotherm is a measure of a highquality surface.

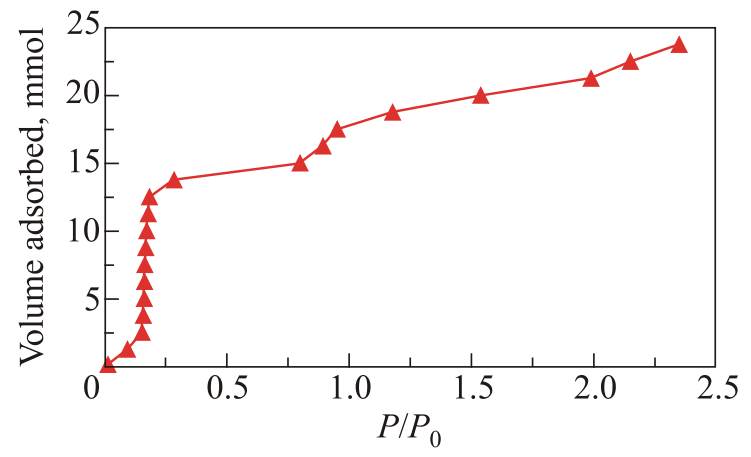

Fig. 2. Experimental isotherm for methane on zeolite 13X. (After Fig. B1 from Ref. 19.) 


\subsection{Adsorbed methane}

Studies of the relaxation of the nuclear spins of methane adsorbed on zeolite $13 \mathrm{X}$ show unexpected features, namely the existence of two prominent peaks in the temperature dependence below $65 \mathrm{~K}$. These peaks are believed to result from the existence of rotational energy levels associated with the tetrahedral molecules, associated with the A, E and $\mathrm{T}$ representations of the tetrahedral group $T_{d}$. The ground state is the A-state with total nuclear spin $I=2$ and the next level is the T-state with $I=1$. The E-state with $I=0$ is not relevant for the nuclear spin relaxation rate. For adsorbed molecules the E-state can split into separate A- and T-states for sites with trigonal symmetry as is the case for molecules near some of the faces in the zeolite cages. The solid red line shown in Fig. 3 is a fit for two distinct T-states using Eq. (10) with heat capacities

$$
\begin{gathered}
C_{A}=\frac{3}{2} N g_{A} k_{B}, \\
C_{T_{i}}=N g_{T_{i}} k_{B}\left[\left(\frac{\Delta_{i}}{2 T}\right)^{2} \operatorname{sech}^{2}\left(\frac{\Delta_{i}}{2 T}\right)\right]
\end{gathered}
$$

for the A- and $\mathrm{T}_{i}$-states, respectively. $g_{A}$ and $g_{T_{i}}$ are the degeneracies of the A- and $\mathrm{T}_{i}$-states, respectively. The best fit is obtained for two T-states with energies $\Delta_{1}=25 \mathrm{~K}$ and $\Delta_{2}=55 \mathrm{~K}$, as represented by the red line in Fig. 3 .

At high temperatures one observes a straightforward thermal activation for the nuclear spin-lattice relaxation times and that is expected for the translational degrees of freedom of the molecules. The best description of the experimental points is obtained using Eq. (6) with a Lorentzian spectral density of the form

$$
J(n \omega)=\frac{\tau}{1+(n \omega \tau)^{2}}
$$

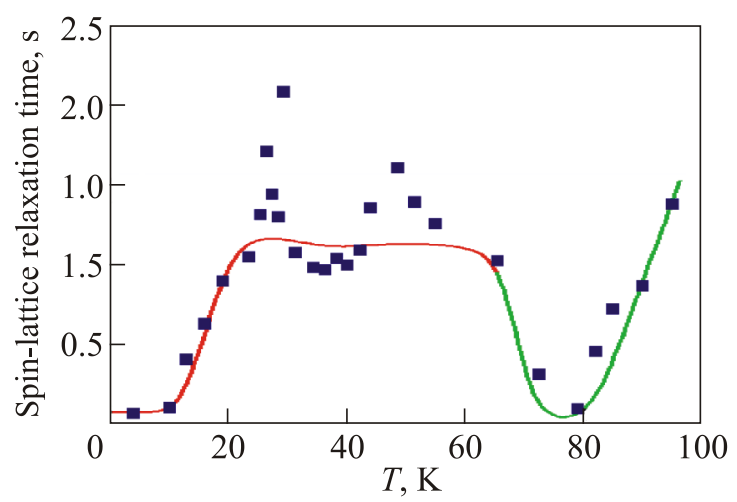

Fig. 3. (Color online) Variation with temperature of the spin-lattice relaxation times of methane adsorbed on zeolite. The solid red line represents the contribution of the Schottky specific heats of the rotational states of methane, and the solid green line is the calculated variation for thermally activated translational motion in the zeolite cage. (Reproduced with permission from Fig. 4 of Ref. 16.) with the correlation time $\tau$ given by the molecular jump time

$$
\tau=\tau_{0} \mathrm{e}^{E_{\text {act }} / T}
$$

with $\tau_{0}=7.1 \cdot 10^{-13} \mathrm{~s}$ and $E_{\text {act }}=(2600 \pm 260) \mathrm{K}$. This fit is given by the green line of Fig. 3. The activation energy compares favorably with the results of Monte Carlo simulations reported by Yashoanth et al. [21]. The rounded minimum at $78 \mathrm{~K}$ in the solid green curve of Fig. 3 is the BPP minimum [22] that arises when the Larmor frequency and the molecular motional frequencies are equal.

\subsection{HD on zeolite $13 X$ and Z-MOF}

The dynamics of HD molecules has been studied for two types of structures, zeolite $13 \mathrm{X}$ and the metal organic framework Z-MOF. HD was explored rather than $\mathrm{H}_{2}$ because of the difficulties associated with the ortho-para conversion of $\mathrm{H}_{2}$ and consequent uncertainty of the ortho$\mathrm{H}_{2}$ concentration as a sample ages. The nuclear relaxation data for HD is also more straightforward to interpret because only translational degrees of freedom are relevant.

At high temperatures we see a simple thermal activation of the translational diffusion of the molecules as shown in Fig. 4. The solid red line of Fig. 4 is found from Eq. (9) with $\tau_{c}=\tau_{0} \mathrm{e}^{E_{\text {act }} / T}, \tau_{0}=6.7 \cdot 10^{-13} \mathrm{~s}$ and $E_{\text {act }}=(73 \pm 3) \mathrm{K}$. These values for $\tau_{c}$ correspond to a translational diffusion constant of $D=(8.2 \pm 2.8) \cdot 10^{-6} \mathrm{~cm}^{2} / \mathrm{s}$ at $T=19.5 \mathrm{~K}$ which is comparable to the values observed by Coulomb et al. [23] using neutron scattering techniques.

One of the unexpected results of the studies was the observation of peaks in the dependence of the nuclear spin-lattice relaxation times at low temperatures for HD on zeolite. This behavior is illustrated in Fig. 5. These peaks suggest that there is a discreet set of energy levels for the translational motion and that these levels contrib-

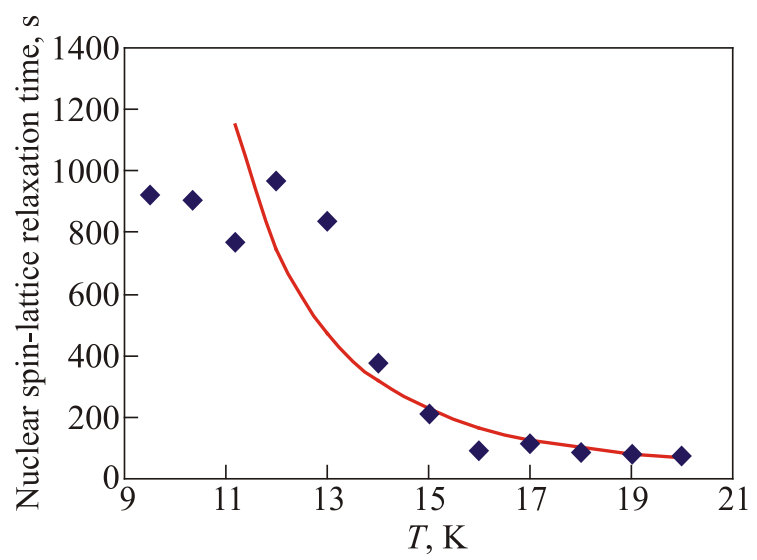

Fig. 4. Dependence of the nuclear spin-spin relaxation times with temperature for HD adsorbed on zeolite 13X. The solid line corresponds to a calculated variation for a thermal activation energy of (72 \pm 3$)$ K. (After Fig. 9 of Ref. 19.) 


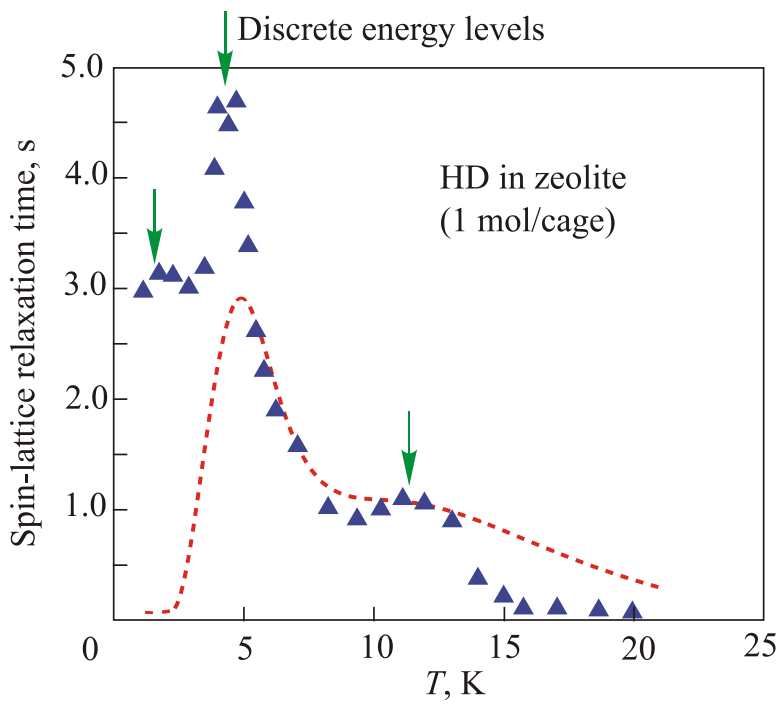

Fig. 5. Observed variation of the nuclear spin-lattice relaxation times of HD in zeolite $13 \mathrm{X}$ at low temperatures. The broken line shows the Schottky contribution for energy levels at 7.5 and 19.6 K. (Reproduced with permission from Fig. 3 of Ref. 17.)

ute to peaks in the heat capacity. From Eq. (6) we expect a corresponding set of peaks in the values of $T_{1}$ at the temperatures corresponding to those energies. For a particle in a potential well, the energy levels are found to be $E_{n, l}=\hbar^{2} k_{n, l}^{2} / 2 m d$, where the $k_{n, l}$ are the spherical Bessel function, $j^{l}(k r)$ [24]. The effective value of $d$ is the diameter of the cage minus twice the hard core radius of the molecule. The lowest energy levels are found to be 1.9, 4.4, 5.6, $7.4 \mathrm{~K}[18]$.

\section{Conclusion}

Studies of the nuclear spin relaxation times of molecules adsorbed in mesoporous structures can provide direct noninvasive measurements of the translational and rotational dynamics of the molecules constrained to the pores of the structures. The activation energies observed for both HD and $\mathrm{CH}_{4}$ measurements appear to be in reasonably good agreement with results reported from studies using other techniques. The most unusual feature was the observation of relatively sharp peaks in the relaxation times at low temperatures for the translational energies. These peaks are close to the energy values expected for the quantization of the translational motion for a particle in the pores, but further studies are needed to verify this interpretation.

\section{Acknowledgments}

This research was supported in part by the National Science Foundation through the award DMR-1303599. We also gratefully acknowledge support from the electronics and machine shops of the Department of Physics at the University of Florida.
1. A. Züttel, Materials Today 6, 24 (2003).

2. D.P. Broom, Hydrogen Storage Materials: Characterization of their Properties, Springer, SBM (2011).

3. G. Chen, L.N. Zakharov, M.E. Bowden, A.J. Karkamkar, S.M. Whittemore, E.B. Garner, T.C. Mikulas, D.A. Dixon, T. Autrey, and S.-Y. Liu, J. Amer. Chem. Soc. 137, 134 (2015).

4. T.A. Makal, J.-R. Li, W. Lu, and H.-C. Zhou, Chem. Soc. Rev. 41, 7761 (2012).

5. C.M. Simon, J. Kim, D.A. Gŏmez-Gualdrŏn, J.S. Camp, Y.G. Chung, R.L. Martin, R. Mercado, M.W. Deem, D. Gunter, M. Haranczyk, D.S. Sholl, R.Q. Snurr, and B. Smit, Energy Environ. Sci. 8, 1190 (2015).

6. D.A. Gómez-Gualdrón, C.E. Wilmer, O.K. Farha, J.T. Hupp, and R.Q. Snurr, J. Phys. Chem. C 118, 6941 (2014).

7. A.V. Vakhrushev and M.V. Suyetin, Nanotechnology 20, 125602 (2009).

8. M. Bienfait, P. Zeppenfeld, N. Dupont-Pavlovsky, M. Muris, M. Johnson, T. Wilson, M.D. Pies, and O. Vilches, Physica B: Condensed. Matter 350, E423 (2004), Proc. Third European Conf. on Neutron Scattering.

9. G. Bomchil, A. Hüller, T. Rayment, S.J. Roser, M.V. Smalley, R.K. Thomas, J.W. White, and A.D. Buckingham, Philos. Trans. Royal Soc. Ln. Series B, Biological Sciences 290, 537 (1980).

10. M.K. Rana, H.S. Koh, H. Zuberi, and D.J. Siegel, J. Phys. Chem. C 118, 2929 (2014).

11. E. Poirier and A. Dailly, Nanotechnology 20, 204006 (2009).

12. C.O. Aren, S. Chavan, C.P. Cabello, E. Garrone, and G.T. Palomino, Chem. Phys. Chem. 11, 3237 (2010).

13. A. Macario, A. Katovic, G. Giordano, F. Iucolano, and D. Caputo, Microporous Mesoporous Mater. 81, 139 (2005).

14. C. Xu and N. Hedin, Materials Today 17, 397 (2014).

15. S.-H. Liu, W.-C. Hsiao, and W.-H. Sie, Adsorption 18, 431 (2012).

16. Y. Ji, J.A. Hamida, and N.S. Sullivan, J. Low Temp. Phys. 158, 509 (2010).

17. Y. Ji, N.S. Sullivan, and J.A. Hamida, J. Low Temp. Phys. 162, 121 (2011).

18. Y. Ji, Y. Tang, J.A. Hamida, and N.S. Sullivan, J. Phys.: Conf. Ser. 400, 012024 (2012).

19. Y. Ji, N.S. Sullivan, Y. Tang, and J.A. Hamida, Materials 6 , 2464 (2013).

20. B.P. Cowan, Nuclear Magnetic Resonance and Relaxation, Cambridge University Press, Cambridge, UK (1997).

21. S. Yashonanth, J.M. Thomas, K. Nowak, and A.K. Cheetham, Nature 331, 601 (1988).

22. N. Bloembergen, E.M. Purcell, and R.V. Pound, Phys. Rev. 73, 679 (1948).

23. J.P. Coulomb, N. Flouquet, N. Dufau, P. Llewellyn, and G. Andre, Microporous Mesoporous Mater. 101, 271 (2007).

24. S. Gasiorowicz, Quantum Physics, John Wiley and Sons, New York (1974). 